\title{
Augmented reality navigation for cranial biopsy and external ventricular drain insertion
}

\author{
*Simon Skyrman, MD, ${ }^{1}$ Marco Lai, MSc,,,3 Erik Edström, MD, PhD, ${ }^{1}$ Gustav Burström, MD, PhD, ${ }^{1}$ \\ Petter Förander, MD, PhD, ${ }^{1}$ Robert Homan, BSc, ${ }^{4}$ Flip Kor, MSc, ${ }^{5}$ Ronald Holthuizen, MSc, ${ }^{4}$ \\ Benno H. W. Hendriks, PhD, ${ }^{2,5}$ Oscar Persson, MD, PhD, ${ }^{1}$ and Adrian Elmi-Terander, MD, PhD ${ }^{1}$
}

\begin{abstract}
1Department of Neurosurgery, Karolinska University Hospital, and Department of Clinical Neuroscience, Karolinska Institutet, Stockholm, Sweden; Philips Research, High Tech Campus 34, Eindhoven; ${ }^{3}$ Eindhoven University of Technology (TU/e), Eindhoven; ${ }^{4}$ Philips Healthcare, Best; and ${ }^{5}$ Department of Biomechanical Engineering, Delft University of Technology, Delft, The Netherlands
\end{abstract}

OBJECTIVE The aim of this study was to evaluate the accuracy (deviation from the target or intended path) and efficacy (insertion time) of an augmented reality surgical navigation (ARSN) system for insertion of biopsy needles and external ventricular drains (EVDs), two common neurosurgical procedures that require high precision.

METHODS The hybrid operating room-based ARSN system, comprising a robotic C-arm with intraoperative conebeam CT (CBCT) and integrated video tracking of the patient and instruments using nonobtrusive adhesive optical markers, was used. A 3D-printed skull phantom with a realistic gelatinous brain model containing air-filled ventricles and 2-mm spherical biopsy targets was obtained. After initial CBCT acquisition for target registration and planning, ARSN was used for 30 cranial biopsies and 10 EVD insertions. Needle positions were verified by CBCT.

RESULTS The mean accuracy of the biopsy needle insertions $(n=30)$ was $0.8 \mathrm{~mm} \pm 0.43 \mathrm{~mm}$. The median path length was $39 \mathrm{~mm}$ (range 16-104 mm) and did not correlate to accuracy $(p=0.15)$. The median device insertion time was 149 seconds (range 87-233 seconds). The mean accuracy for the EVD insertions ( $n=10)$ was $2.9 \mathrm{~mm} \pm 0.8 \mathrm{~mm}$ at the tip with a $0.7^{\circ} \pm 0.5^{\circ}$ angular deviation compared with the planned path, and the median insertion time was 188 seconds (range 135-400 seconds).

CONCLUSIONS This study demonstrated that ARSN can be used for navigation of percutaneous cranial biopsies and EVDs with high accuracy and efficacy.

https://thejns.org/doi/abs/10.3171/2021.5.FOCUS20813

KEYWORDS surgical navigation; image-guided surgery; augmented reality; neurosurgery; brain biopsy; external ventricular drainage

$\mathrm{S}$ URGICAL navigation has played an important role in improving accuracy and reducing invasiveness in modern neurosurgery and is today considered the standard of care in a wide array of neurosurgical procedures. ${ }^{1-4}$ In intracranial tumor surgery, the use of navigation results in lower complication rates, maximized extent of resection, and improved patient prognosis. ${ }^{5,6}$ Frameless techniques for intracranial biopsy have been developed to improve patient comfort and clinical workflow compared with the frame-based stereotactic technique., ${ }^{7}$

Even though surgical navigation systems have continuously improved since their introduction in the early 1990s, the principal technology has remained essentially unchanged. ${ }^{9}$ In principle, the technique allows the surgeon to track a surgical instrument in three dimensions and in relation to the patient's anatomy, based on coregistration with a dynamic reference frame and preoperative $\mathrm{CT}$ or MRI. ${ }^{10}$

During the last decade, however, augmented reality (AR) has emerged as a promising and significant technical advancement in surgical navigation. ${ }^{11}$ AR has been successfully applied in several types of neurosurgical procedures, ${ }^{12-15}$ including frame-based intracranial biopsy. ${ }^{16} \mathrm{AR}$ can be defined as the overlay of computer-generated visual

ABBREVIATIONS AR = augmented reality; $\mathrm{ARSN}=\mathrm{AR}$ surgical navigation; $\mathrm{CBCT}=$ cone-beam CT; EVD = external ventricular drain; OR = operating room; OTS = optical tracking system; PLA = polylactic acid; $\mathrm{PVA}=$ polyvinyl alcohol; $w \mathrm{t} \%=$ weight percentage.

SUBMITTED September 11, 2020. ACCEPTED May 17, 2021.

INCLUDE WHEN CITING DOI: 10.3171/2021.5.FOCUS20813.

* S.S. and M.L. contributed equally to this work. 


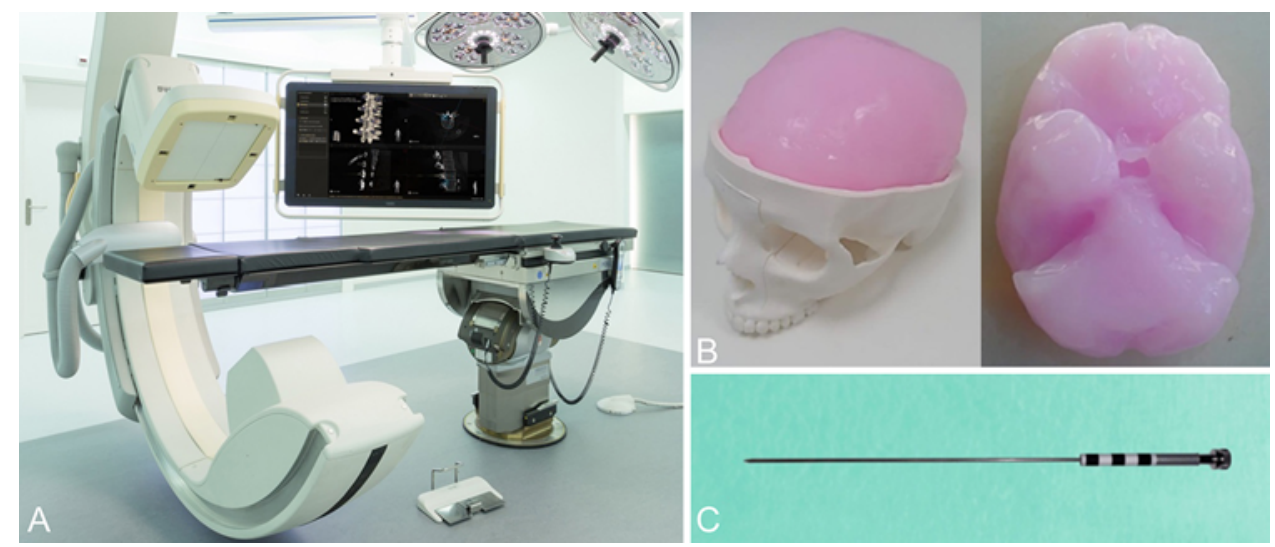

FIG. 1. A: The hybrid OR-based ARSN system with a ceiling-mounted robotic C-arm for CBCT acquisition and registration. An OTS with 4 optical video cameras is embedded in the flat panel detector of the $\mathrm{C}$-arm and is used for optical tracking of instruments and the patient. Once the registration is completed, the $\mathrm{C}$-arm can be removed and the tracking switched to a separate, freestanding OTS during surgical procedures. B: The 3D-printed skull phantom and realistic gelatinous brain model containing air-filled ventricles and 2-mm spherical biopsy targets. C: The needle used for biopsies and EVD insertions. A cylindrical optical marker consisting of alternating black-and-white stripes was attached around the proximal part of the needle to allow tracking of the instrument.

information on real-world objects. By overlaying anatomical objects as 3D virtual models on the view of the surgical field, real-time visualization of surgical targets or risk organs is possible. The navigational data can be projected directly onto the surgical field ${ }^{17}$ or displayed on a video stream of the surgical field, ${ }^{18}$ either on a separate screen or in a head-mounted display (HMD) device. However, a recent review of AR technology indicates that the majority of publications concern microscope-based systems. ${ }^{11}$

An ideal AR navigation system should avoid a complicated or time-consuming setup and registration, be easy to use, and have an AR display independent of the microscope, when the surgical procedure so requires..$^{11}$ It should also allow visualization and augmentation of the anatomy without distracting the surgeon or obscuring the surgical field. ${ }^{19}$ Finally, the system must be accurate as well as offer the possibility of confirming the results with intraoperative imaging. ${ }^{20,21}$

In an effort to meet these requirements, we have adapted and evaluated a novel AR surgical navigation (ARSN) system. The system has successfully been used in spine surgery, ${ }^{12,22,23}$ and an application for endoscopic skull base surgery is under development. ${ }^{18}$ The ARSN system relies on intraoperative cone-beam CT (CBCT) and live video, in combination with intraoperatively placed skin markers for registration and patient tracking. ${ }^{12,23,24}$ The augmented video feed is displayed on a separate monitor. As a first step in adapting the system for use in cranial neurosurgery, an AR navigation application for intracranial biopsy and placement of external ventricular drains (EVDs) has been developed. These are common and highly standardized neurosurgical procedures with a high demand for accuracy, and thus are suitable for evaluating the performance of a navigation system. In this study, we present and evaluate the ARSN system applied for cranial biopsy and EVD insertion. The aim of this study was to measure the technical accuracy of the ARSN system in terms of deviation from the target or the planned path in millimeters.
Furthermore, the concept of AR navigation for these procedures, in terms of insertion time and surgical workflow, was evaluated.

\section{Methods \\ The ARSN System}

The ARSN system used in this study has been previously described. ${ }^{25,26}$ It is a hybrid operating room (OR)based navigation system with a ceiling-mounted robotic C-arm (Allura FlexMove, Philips Healthcare), with intraoperative CBCT capability (Fig. 1A). An optical tracking system (OTS) with four optical video cameras embedded in the flat panel detector of the C-arm is used for coregistration, patient and instrument tracking, and image augmentation. Flat adhesive optical markers, recognized by the OTS, are applied to the skin or the Mayfield clamp and are used for coregistration as well as patient position tracking. Using the C-arm, a CBCT is performed to automatically coregister the patient 3D image and patient position. Once the coregistration is completed the $\mathrm{C}$-arm can be removed and the tracking switched to a separate, freestanding OTS during surgical procedures. The system's own proprietary software is used for segmentation of anatomical structures of interest and planning of paths to targets. A video stream of the surgical field, augmented with the segmented anatomical structures, tracked instruments, and planned paths, is displayed on a dedicated medical monitor.

\section{The Skull and Brain Phantom}

A high-quality CT scan (0.75-mm slice thickness) obtained in an anonymous adult female patient downloaded from an online DICOM library ${ }^{27}$ was used as a template for the skull phantom and modified to allow detachment of the calvaria (Fig. 1B). The skull was 3D printed in polylactic acid with calcium (PLA $+\mathrm{CaCO}_{3}$ ), resulting in a 

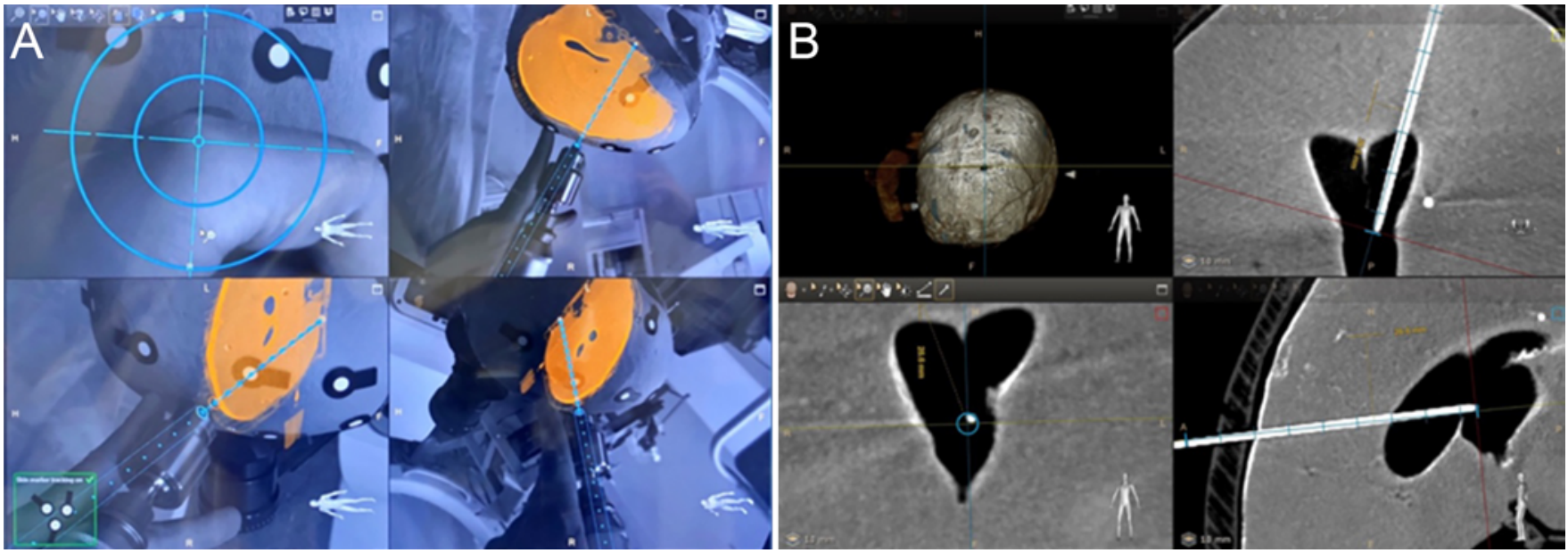

FIG. 2. A: Screenshot of the ARSN system display during drilling of the skull phantom. A bull's-eye view in the axis of the planned path (upper left) as well as augmented images of the axial, coronal, and sagittal planes are displayed to guide the surgeon during the procedure. In this case, the target was located at the base of the skull of the patient's right side. B: Screenshot of the ARSN monitor display of an EVD placed in a good position in the ventricular system of the brain phantom. The EVD catheter follows the planned path with minimal angular deviation and is located intraventricularly in the frontal horn of the ipsilateral lateral ventricle with its tip in the area of the foramen of Monro.

bone-like CT attenuation, higher than that of PLA alone (480 HU vs $25 \mathrm{HU}$ ). A 3D computational model of the brain was segmented from the same CT scan and reduced by $4 \mathrm{~mm}$ in every direction to fit the skull. The negative image of the brain was 3D printed and used as a mold for the brain phantom. Air-filled ventricles were created by printing the ventricular system in PLA that could be washed out after molding of the brain. The brain tissue model was created using a mixture of 6 weight percentage (wt\%) polyvinyl alcohol (PVA; Sekisui SELVOL Polyvinyl Alcohol 165), $56 \mathrm{wt} \%$ water, $38 \mathrm{wt} \%$ coolant, and $1 \mathrm{wt} \%$ of barium sulfate $\left(\mathrm{BaSO}_{4}\right.$; Sigma-Aldrich), using one freeze-thaw cycle at $-25^{\circ} \mathrm{C}$. The use of a coolant prevented the 3D-printed mold from breaking due to expansion of the PVA mixture during freezing. Barium sulfate was used to mimic the CT attenuation of the brain; $1 \mathrm{wt} \%$ resulted in $126 \mathrm{HU}$, slightly higher than normal brain attenuation $(40 \mathrm{HU})$. The stiffness of the material could be controlled by repeated freeze-thaw cycles and the concentration of PVA. ${ }^{28}$ The mechanical test on the PVA material showed that the elastic modulus of the brain model was higher than that of the real brain tissue. After the brain was created, 30 steel balls ( $2 \mathrm{~mm} \pm 5 \mu$ m diameter) were placed at several depths inside the brain phantom, serving as targets for the biopsy experiments.

\section{Biopsy and EVD Insertion}

All insertions were performed by a senior consultant neurosurgeon with 10 years of neurosurgical experience. The skull phantom was fixed in a Mayfield clamp. Flat adhesive skin markers for optical tracking were attached to the skull and the clamp. A CBCT was acquired for registration and planning. Using the proprietary software of the ARSN system, the 2-mm-diameter bearing balls in the gelatinous brain model were assigned as targets and biopsy paths were planned. A sharp, 188-mm-long, 1.5-mm- diameter stainless steel biopsy needle was tracked in three dimensions by the navigation system and used for insertions. To enable optical tracking, a 30-mm-long, 5-mmdiameter cylindrical optical marker, consisting of alternating 5-mm black-and-white stripes, was attached around the proximal part of the needle and calibrated with the navigation system (Fig. 1C). A standard handheld surgical bone drill was used to create a 3-mm-diameter hole in the skull phantom. Identification of the entry point and angle of drilling, as well as the needle insertion, were guided by the ARSN system. An augmented visualization of the path overlaid on the video of the surgical field was displayed together with the tracked needle depicted in the axial, sagittal, and coronal projections of the acquired intraoperative CBCT (Fig. 2A and Video 1).

VIDEO 1. Video presentation of the simulated ARSN biopsy procedure. VR = virtual reality. Copyright Simon Skyrman. Published with permission. Click here to view.

After the needle was positioned, a new CBCT was acquired for verification of the needle position and accuracy measurements. Thirty individual biopsy insertions were performed. The same needle and technique were used to perform 10 EVD insertions, of which 6 were placed frontally with entry at Kocher's point and the planned tip position in the frontal horn of the ipsilateral lateral ventricle at the foramen of Monro, and 4 were placed occipitally with entry at Frazier's point and the planned tip position in the body of the ipsilateral lateral ventricle.

\section{Accuracy and Time Measurements}

Accuracy measurements were performed by a blinded senior consultant neurosurgeon on postprocedure scans. The simulated biopsy accuracy was defined as the distance between the tip of the inserted needle and the center of the target (i.e., the bearing ball). The CBCT images were reconstructed so that the tip of the needle and the 

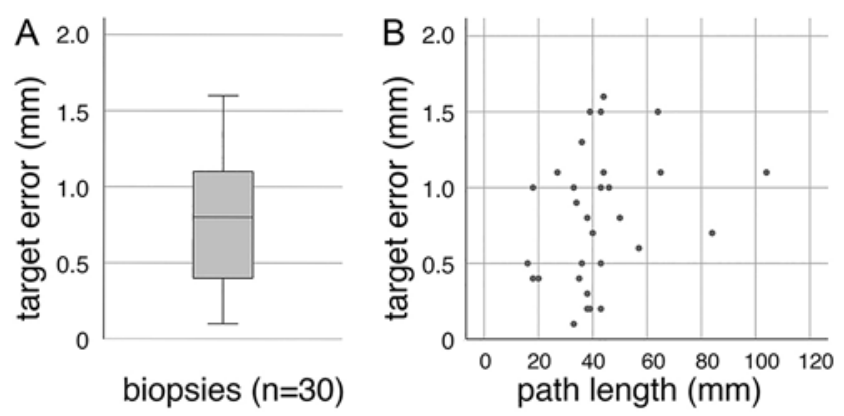

FIG. 3. A: Boxplot presenting the accuracy achieved in the AR-guided biopsy procedures $(n=30)$. The target error was defined as the distance in millimeters from the biopsy needle tip to the center of the biopsy target, a 2-mm steel bearing ball. The boxplot displays the median as a solid line, hinges correspond to upper and lower quartiles, and whiskers indicate data up to a 1.5 interquartile range. B: Scatterplot presenting each individual biopsy target error ( $y$-axis) plotted as a function of path length (x-axis), showing no correlation between the two parameters ( $p$ $=0.15)$.

center of the target were in the same plane, after which the distance was calculated by measuring from the tip of the needle to the center of the bearing ball, with subtraction of the ball radius of $1 \mathrm{~mm}$. For the simulated EVD insertions, the accuracy was similarly defined as the distance from the tip of the inserted needle to the end of the planned path. In addition, the angular deviation of the inserted needle in relation to the predefined path was measured in the insertion plane. The rationale behind reporting accuracy differently for biopsy and EVD was based on clinical considerations. Different trajectories may be used for reaching a biopsy target in the brain. The important accuracy is in relation to the target, measured as the distance between the needle and the target, rather than the entry point and the angulation of the chosen path. An anatomically correct EVD position (Fig. 2B), however, requires adherence to a path defined by an anatomical starting point, while the tip placement has a larger degree of freedom, as long as it is in the area of the foramen of Monro. Thus, accurate EVD placement is not dependent on millimeter accuracy at the tip but rather on the correct angulation of the EVD and adherence to the planned path. Therefore, both accuracy at the tip and the angular deviation of the placed EVD with respect to the planned path were measured. ${ }^{22}$ The time for each simulated biopsy and EVD insertion was recorded from the moment the surgeon first started identifying the entry point until the biopsy needle or EVD was in the intended position.

\section{Statistical Analysis}

Accuracy measurement, angular deviations, and insertion times were expressed as mean \pm standard deviation and as median (range). Pearson's product moment correlation coefficient was used for testing correlation. Statistical analysis was performed using SPSS version 12.0 (SPSS Inc.), and a $\mathrm{p}$ value $<0.05$ was considered significant.

\section{Results}

The median accuracy for the simulated biopsies $(\mathrm{n}=$
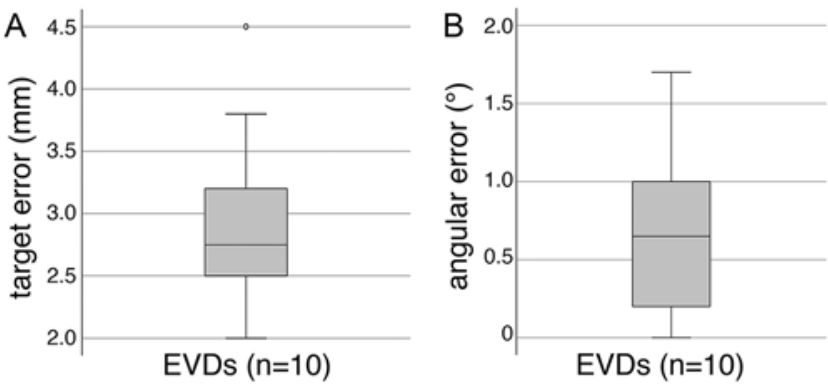

FIG. 4. A: Boxplot presenting EVD placement accuracy defined as the distance from the planned path end to the tip of the needle inserted, measured on postinsertion CBCT images. B: Boxplot presenting EVD placement accuracy defined as angular deviation from the preplanned EVD path in degrees. The angular error, defined as the angle between the predefined path and the axis of symmetry of the inserted needle, was measured in the insertion plane.

30) was $0.8 \mathrm{~mm}$ (range $0.1-1.6 \mathrm{~mm}$; mean $0.8 \pm 0.43 \mathrm{~mm}$ ) as presented in the boxplot in Fig. 3A. The median navigation time following the planned path was 149 seconds (range 87-233 seconds). The median path length was 39 $\mathrm{mm}$ (range 16-105 $\mathrm{mm}$ ) and did not correlate with accuracy $(\mathrm{p}=0.15)$ (Fig. 3B). The median accuracy for EVD insertions $(\mathrm{n}=10)$ was $2.8 \mathrm{~mm}$ (range $2.0-4.5 \mathrm{~mm}$; mean $2.9 \mathrm{~mm} \pm 0.8 \mathrm{~mm}$ ) at the tip with a $0.7^{\circ} \pm 0.5^{\circ}$ angular deviation. The results for EVD insertions are depicted in Fig. 4. All EVDs had a good intraventricular position (Fig. 2B), and the median navigation time was 188 seconds (range 135-400 seconds).

\section{Discussion}

\section{Accuracy}

In this study, we present the development of an ARSN system for cranial biopsies and EVD insertions. The system was tested on a skull phantom with a realistic gelatinous brain model. Submillimeter accuracy for simulated intracranial biopsies was achieved, and the EVDs were placed with insignificant divergence from the planned paths. The mean accuracy of $0.8 \mathrm{~mm}$ achieved in this study is comparable to the results of previously described frameless stereotactic setups (Table 1). ${ }^{29-33}$ The accuracy for EVD insertions was slightly lower $(2.9 \mathrm{~mm})$, which is likely explained by the fact that the intraventricular target areas were less well defined than the bearing balls. In the clinical situation, a biopsy requires submillimeter accuracy, while the tolerance in EVD insertions is much greater. ${ }^{34}$ In this study, all insertions were performed freehand, guided by AR navigation on an external monitor. The use of a dedicated high-definition, ceiling-mounted monitor allowed for AR images to be easily displayed and used for accurate navigation (Fig. 2A and B). Even though CTguided freehand biopsies of deep-seated lesions have been proven to be safe, it is reasonable to assume that fixation of the needle during the procedure is safer and more effective..$^{35}$ The ARSN system presented in this study can be combined with a surgical or robotic arm, ${ }^{36,37}$ or any other type of biopsy needle holding device, for needle stabilization and increased safety. 
TABLE 1. Accuracy of comparable, frameless stereotactic biopsy systems as presented in previous studies and the current study

\begin{tabular}{lccc}
\hline \multicolumn{1}{c}{ Authors \& Year } & $\begin{array}{c}\text { Mean Accuracy } \\
\pm \text { SD }(\mathrm{mm})\end{array}$ & $\begin{array}{c}\text { Frameless Stereotactic } \\
\text { Biopsy System }\end{array}$ & $\begin{array}{c}\text { Sample } \\
\text { Size (n) }\end{array}$ \\
\hline Dorward et al., 1999 ${ }^{30}$ & $1.3 \pm 0.6$ & EasyGuide Neuro system (Philips Medical Systems Nederland BV, Best, the Netherlands) & 258 \\
\hline Brommeland \& Hennig, 200029 & $2.9 \pm 0.2$ & The Viewing Wand (Elekta Instrument AB, Stockholm, Sweden) & 60 \\
\hline $\begin{array}{l}\text { Quiñones-Hinojosa et al., } \\
2006^{31}\end{array}$ & $1.0 \pm 0.3$ & $\begin{array}{c}\text { StealthStation Treatment Guidance System (Medtronic Sofamor Danek, Minneapolis, } \\
\text { Minnesota) }\end{array}$ \\
\hline Ringel et al., 200932 & $0.7 \pm 0.7$ & VarioGuide (Brainlab AG, Feldkirchen, Germany) & 4 \\
\hline Widmann et al., 200833 & $1.5 \pm 0.8$ & Vertek aiming device (Medtronic Inc., Louisville, Colorado) & 129 \\
\hline Current study & $0.8 \pm 0.43$ & ARSN system (Philips Medical Systems Nederland BV, Best, the Netherlands) \\
\hline
\end{tabular}

\section{Advantages of the ARSN Setup}

The system is based on a hybrid OR solution providing a number of benefits. ${ }^{38}$ The integration of video cameras in the ceiling-mounted $\mathrm{C}$-arm simplifies the registration process. Once the initial CBCT scan is performed, the patient is automatically coregistered. ${ }^{25}$ Compared with other frameless techniques, the ARSN system could have a possible advantage for deep-seated lesions, as navigation systems dependent on registration of anatomical surface structures may lessen in accuracy with increasing distance from the face. ${ }^{39}$ This phenomenon is likely explained by small angular inaccuracies in surface-based coregistration, which will cause an amplification of the absolute error the farther from the coregistration surface the target gets. The CBCT-based registration protocol used by the ARSN system, on the other hand, has a homogeneously distributed accuracy that is independent of the target depth or fixation of the head (Fig. 3B). ${ }^{12,22}$

In addition, while the intraoperative $\mathrm{CBCT}$ capability of the ARSN system is used for patient imaging and coregistration, it can also provide the surgeon with fully updated intraoperative images if needed. A contrast-enhanced CBCT can be performed for verification of biopsy position and exclusion of early postoperative hemorrhage immediately after the biopsy in the OR. ${ }^{40-42}$ This early postoperative CBCT could replace other postoperative radiological examinations, making the procedure even more efficient. The average radiation dose from one CBCT is approximately $50 \%(1 \mathrm{mSv})$ of a standard diagnostic CT scan $(2 \mathrm{mSv}){ }^{43}$ Hence, even if two CBCT sessions are performed for planning and verification, the total radiation dose will not exceed that of one conventional postoperative diagnostic CT scan. Moreover, the use of intraoperative CBCT has the potential to compensate for brain shift, addressing one of the major limitations of current navigation systems..$^{44,45}$

\section{Time Aspects}

Based on the time measurements for insertions in this study, and previous experience of the system in clinical applications, ${ }^{12}$ the estimated accumulated time for CBCT acquisition, navigation, and needle insertion would be less than 9 minutes. In this study, planning of trajectories was performed for multiple targets and cannot fully represent the considerations in a real surgical case. However, in a real surgical scenario, preplanning can be made on preop- erative MRI that is later fused to the intraoperative $\mathrm{CBCT}$ for efficient use of OR time.

\section{Frameless Stereotaxy}

Stereotactic biopsies can be performed in awake or lightly sedated patients using local anesthesia, avoiding the risks of general anesthesia in patients with comorbidities, and reducing the OR time. However, current solutions require fixation of the head in either a stereotactic frame or a head clamp, which, even with application of local anesthetics, may be a painful procedure. In this study, the skull phantom was fixed to the surgical table in a head clamp. However, the ARSN system does not rely on rigid fixation of the head, since tracking of the patient position is performed in real time by the system's video cameras, and patient movements are compensated for. ${ }^{12,22}$ Reducing discomfort for the patient and shortening OR time without sacrificing accuracy could be potential advantages of the ARSN system.

\section{Limitations}

This study was performed on a phantom that, although realistic, is not identical to a real clinical scenario. No real biopsies were obtained, and no real EVDs were placed. Despite the promising results and high accuracy obtained in this setup, further studies on cadaveric and animal models are required to confirm accuracy and safety in as realistic conditions as possible before clinical use could be considered.

\section{Conclusions}

The results of this study indicate that the ARSN system can guide cranial biopsies and EVD insertions with high accuracy and has the potential to improve the workflow for biopsy procedures in a real surgical setting. Further clinical studies are warranted for confirmation of the results presented here.

\section{Acknowledgments}

Mr. Lai has received funding from the European Union's Horizon 2020 research and innovation program under the Marie Skłodowska-Curie grant agreement number 721766 (FBI).

\section{References}

1. Orringer DA, Golby A, Jolesz FJ. Neuronavigation in the sur- 
gical management of brain tumors: current and future trends. Expert Rev Med Devices. 2012;9(5):491-500.

2. Stone SS, Rutka JT. Utility of neuronavigation and neuromonitoring in epilepsy surgery. Neurosurg Focus. 2008; 25(3):E17.

3. Schroeder HW, Wagner W, Tschiltschke W, Gaab MR. Frameless neuronavigation in intracranial endoscopic neurosurgery. J Neurosurg. 2001;94(1):72-79.

4. Holly LT, Foley KT. Intraoperative spinal navigation. Spine (Phila Pa 1976). 2003;28(15)(suppl):S54-S61.

5. Wu JS, Zhou LF, Tang WJ, Mao Y, Hu J, Song YY, et al. Clinical evaluation and follow-up outcome of diffusion tensor imaging-based functional neuronavigation: a prospective, controlled study in patients with gliomas involving pyramidal tracts. Neurosurgery. 2007;61(5):935-949.

6. Wirtz CR, Albert FK, Schwaderer M, Heuer C, Staubert A, et al. The benefit of neuronavigation for neurosurgery analyzed by its impact on glioblastoma surgery. Neurol Res. 2000; 22(4):354-360.

7. Dammers R, Haitsma IK, Schouten JW, Kros JM, Avezaat CJ, Vincent AJ. Safety and efficacy of frameless and framebased intracranial biopsy techniques. Acta Neurochir (Wien). 2008;150(1):23-29.

8. Paleologos TS, Dorward NL, Wadley JP, Thomas DG. Clinical validation of true frameless stereotactic biopsy: analysis of the first 125 consecutive cases. Neurosurgery. 2001;49(4): 830-837.

9. Citardi MJ, Agbetoba A, Bigcas JL, Luong A. Augmented reality for endoscopic sinus surgery with surgical navigation: a cadaver study. Int Forum Allergy Rhinol. 2016;6(5):523-528.

10. Spetzger U, Laborde G, Gilsbach JM. Frameless neuronavigation in modern neurosurgery. Minim Invasive Neurosurg. 1995;38(4):163-166.

11. Meola A, Cutolo F, Carbone M, Cagnazzo F, Ferrari M, Ferrari V. Augmented reality in neurosurgery: a systematic review. Neurosurg Rev. 2017;40(4):537-548

12. Burström G, Buerger C, Hoppenbrouwers J, Nachabe R, Lorenz C, Babic D, et al. Machine learning for automated 3-dimensional segmentation of the spine and suggested placement of pedicle screws based on intraoperative conebeam computer tomography. J Neurosurg Spine. 2019;31(1): 147-154.

13. Carl B, Bopp M, Saß B, Pojskic M, Nimsky C. Augmented reality in intradural spinal tumor surgery. Acta Neurochir (Wien). 2019;161(10):2181-2193.

14. Cabrilo I, Bijlenga P, Schaller K. Augmented reality in the surgery of cerebral aneurysms: a technical report. Neurosurgery. 2014;10(2)(suppl 2):252-261.

15. Guha D, Alotaibi NM, Nguyen N, Gupta S, McFaul C, Yang VXD. Augmented reality in neurosurgery: a review of current concepts and emerging applications. Can J Neurol Sci. 2017;44(3):235-245.

16. Satoh M, Nakajima T, Yamaguchi T, Watanabe E, Kawai K. Application of augmented reality to stereotactic biopsy. Neurol Med Chir (Tokyo). 2019;59(11):444-447.

17. Besharati Tabrizi L, Mahvash M. Augmented reality-guided neurosurgery: accuracy and intraoperative application of an image projection technique. J Neurosurg. 2015;123(1):206211.

18. Lai M, Skyrman S, Shan C, Babic D, Homan R, Edström $\mathrm{E}$, et al. Fusion of augmented reality imaging with the endoscopic view for endonasal skull base surgery; a novel application for surgical navigation based on intraoperative cone beam computed tomography and optical tracking. PLoS One. 2020;15(1):e0227312.

19. Citardi MJ, Yao W, Luong AJ. Next-generation surgical navigation systems in sinus and skull base surgery. Otolaryngol Clin North Am. 2017;50(3):617-632.

20. Nimsky C, Carl B. Historical, current, and future intraopera- tive imaging modalities. Neurosurg Clin N Am. 2017;28(4): 453-464.

21. Willems PW, van der Sprenkel JW, Tulleken CA, Viergever MA, Taphoorn MJ. Neuronavigation and surgery of intracerebral tumours. J Neurol. 2006;253(9):1123-1136.

22. Burström G, Nachabe R, Persson O, Edström E, Elmi Terander A. Augmented and virtual reality instrument tracking for minimally invasive spine surgery: a feasibility and accuracy study. Spine (Phila Pa 1976). 2019;44(15):1097-1104.

23. Elmi-Terander A, Burström G, Nachabe R, Skulason H, Pedersen K, Fagerlund M, et al. Pedicle screw placement using augmented reality surgical navigation with intraoperative 3D imaging: a first in-human prospective cohort study. Spine (Phila Pa 1976). 2019;44(7):517-525.

24. Elmi-Terander A, Burström G, Nachabé R, Fagerlund M, Ståhl F, Charalampidis A, et al. Augmented reality navigation with intraoperative 3D imaging vs fluoroscopy-assisted free-hand surgery for spine fixation surgery: a matchedcontrol study comparing accuracy. Sci Rep. 2020;10(1):707.

25. Edström E, Burström G, Nachabe R, Gerdhem P, Elmi Terander A. A novel augmented-reality-based surgical navigation system for spine surgery in a hybrid operating room: design, workflow, and clinical applications. Oper Neurosurg (Hagerstown). 2020;18(5):496-502.

26. Elmi-Terander A, Burström G, Nachabé R, Fagerlund M, Ståhl F, Charalampidis A, et al. Augmented reality navigation with intraoperative 3D imaging vs fluoroscopy-assisted free-hand surgery for spine fixation surgery: a matchedcontrol study comparing accuracy. Sci Rep. 2020;10(1):707.

27. Fritz J, U-Thainual P, Ungi T, Flammang AJ, Fichtinger G, Iordachita II, Carrino JA. Augmented reality visualisation using an image overlay system for MR-guided interventions: technical performance of spine injection procedures in human cadavers at 1.5 Tesla. Eur Radiol. 2013;23(1):235-245.

28. Chen RK, Shih AJ. Multi-modality gellan gum-based tissuemimicking phantom with targeted mechanical, electrical, and thermal properties. Phys Med Biol. 2013;58(16):5511-5525.

29. Brommeland T, Hennig R. A new procedure for frameless computer navigated stereotaxy. Acta Neurochir (Wien). 2000; 142(4):443-448.

30. Dorward NL, Alberti O, Palmer JD, Kitchen ND, Thomas DG. Accuracy of true frameless stereotaxy: in vivo measurement and laboratory phantom studies. Technical note. $J$ Neurosurg. 1999;90(1):160-168.

31. Quiñones-Hinojosa A, Ware ML, Sanai N, McDermott MW. Assessment of image guided accuracy in a skull model: comparison of frameless stereotaxy techniques vs. frame-based localization. J Neurooncol. 2006;76(1):65-70.

32. Ringel F, Ingerl D, Ott S, Meyer B. VarioGuide: a new frameless image-guided stereotactic system-accuracy study and clinical assessment. Neurosurgery. 2009;64(5)(suppl 2):365373.

33. Widmann G, Eisner W, Kovacs P, Fiegele T, Ortler M, Lang TB, et al. Accuracy and clinical use of a novel aiming device for frameless stereotactic brain biopsy. Minim Invasive Neurosurg. 2008;51(6):361-369.

34. Abdoh MG, Bekaert O, Hodel J, Diarra SM, Le Guerinel $\mathrm{C}$, et al. Accuracy of external ventricular drainage catheter placement. Acta Neurochir (Wien). 2012;154(1):153-159.

35. Wen DY, Hall WA, Miller DA, Seljeskog EL, Maxwell RE. Targeted brain biopsy: a comparison of freehand computed tomography-guided and stereotactic techniques. Neurosurgery. 1993;32(3):407-413.

36. Burström G, Balicki M, Patriciu A, Kyne S, Popovic A, Holthuizen R, et al. Feasibility and accuracy of a robotic guidance system for navigated spine surgery in a hybrid operating room: a cadaver study. Sci Rep. 2020;10(1):7522.

37. Balicki M, Kyne S, Toporek G, Holthuizen R, Homan R, Popovic A, et al. Design and control of an image-guided ro- 
bot for spine surgery in a hybrid OR. Int J Med Robot. 2020; 16(4):e2108.

38. Casar Berazaluce AM, Hanke RE, von Allmen D, Racadio JM. The state of the hybrid operating room: technological acceleration at the pinnacle of collaboration. Curr Surg Rep. 2019;7(4):7.

39. Wang MN, Song ZJ. Properties of the target registration error for surface matching in neuronavigation. Comput Aided Surg. 2011;16(4):161-169.

40. Schafer S, Wang A, Otake Y, Webster Stayman J, Zbijewski W, Kleinszig G, et al. Intraoperative imaging for patient safety and QA: detection of intracranial hemorrhage using C-arm cone-beam CT. Proc SPIE. 2013;8671:86711X.

41. Lee S, Gallia GL, Reh DD, Schafer S, Uneri A, Mirota DJ, et al. Intraoperative $\mathrm{C}$-arm cone-beam computed tomography: Quantitative analysis of surgical performance in skull base surgery. Laryngoscope. 2012;122(9):1925-1932.

42. Wang A, Stayman J, Otake Y, Gallia G, Siewerdsen J. Lowdose C-arm cone-beam CT with model-based image reconstruction for high-quality guidance of neurosurgical intervention. Med Phys. 2014;41(6 pt 30):526.

43. Wang C, Nguyen G, Toncheva G, Jiang X, Ferrell A, Smith $\mathrm{T}$, Yoshizumi T. Evaluation of patient effective dose of neurovascular imaging protocols for $\mathrm{C}$-arm cone-beam CT. AJR Am J Roentgenol. 2014;202(5):1072-1077.

44. Smit-Ockeloen I, Ruijters D, Breeuwer M, Babic D, Brina O, Pereira VM. Accuracy assessment of CBCT-based volumetric brain shift field. In: Oyarzun Laura C, Shekhar R, Wesarg $\mathrm{S}$, et al, eds. Clinical Image-Based Procedures. Translational Research in Medical Imaging. CLIP 2015. Lecture Notes in Computer Science. Vol 9401. Springer; 2015:1-9.

45. Bayer S, Ravikumar N, Strumia M, et al. Intraoperative brain shift compensation using a hybrid mixture model. In: Frangi A, Schnabel J, Davatzikos C, et al, eds. Medical Image Computing and Computer Assisted Intervention - MICCAI 2018. MICCAI 2018. Lecture Notes in Computer Science. Vol 11073. Springer; 2018:116-124.

\section{Disclosures}

Karolinska University Hospital and Philips Healthcare have a major collaboration agreement. The authors affiliated with Philips Research and Philips Healthcare (M.L., R.H., R.H., and B.H.W.H.) have financial interests in the subject matter, materials, and equipment, in the sense that they are employees of Philips. Philips provided support in the form of salaries.

\section{Author Contributions}

Conception and design: Skyrman, Lai, Edström, Burström, Homan, Kor, Holthuizen, Hendriks, Persson, Elmi-Terander. Acquisition of data: Skyrman, Lai, Homan, Holthuizen, Hendriks, Persson, Elmi-Terander. Analysis and interpretation of data: Skyrman, Edström, Persson, Elmi-Terander. Drafting the article: Skyrman. Critically revising the article: Skyrman, Edström, Burström, Förander, Hendriks, Persson, Elmi-Terander. Reviewed submitted version of manuscript: all authors. Approved the final version of the manuscript on behalf of all authors: Skyrman. Statistical analysis: Skyrman, Elmi-Terander. Administrative/ technical/material support: Skyrman, Lai, Homan, Kor, Holthuizen, Persson. Study supervision: Edström, Förander, Persson, Elmi-Terander.

\section{Supplemental Information \\ Videos}

Video 1. https://vimeo.com/558663534.

\section{Correspondence}

Simon Skyrman: Karolinska Institutet, Stockholm, Sweden. simon.skyrman@sll.se. 